Original article

\title{
End-stage kidney disease in Sidi Bel Abbes, Algeria: Epidemiological profile of hemodialysis patients from 2015 to 2018
}

\author{
Katia Berkache ${ }^{\mathrm{a}}$, Zohra Bengharez ${ }^{\mathrm{a}, *}$, Bastien Poitier ${ }^{\mathrm{b}}$, Djamila Ouabdesslam ${ }^{\mathrm{c}}$, \\ Abdelkrim Guerinik ${ }^{\mathrm{d}}$, Mourad Amrane ${ }^{\mathrm{b}}$

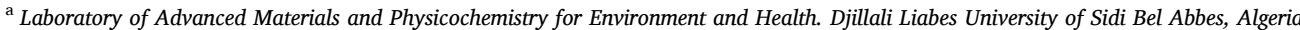 \\ ${ }^{\mathrm{b}}$ University of Paris, Cardiac Surgery Department, AH-HP, Georges Pompidou European Hospital, F-75015 Paris, France \\ ${ }^{\mathrm{c}}$ Epidemiology Department, University Hospital Hassani Abdelkader, Sidi Bel Abbes, Algeria \\ ${ }^{\mathrm{d}}$ Hemodialysis Clinic El Wissam, Sidi Bel Abbes, Algeria
}

\section{A R T I C L E I N F O}

\section{Keywords:}

Hemodialysis

ESKD

Epidemiological profile

Prevalence

Sidi Bel Abbes

\begin{abstract}
A B S T R A C T
Objectives: End-stage kidney diseases (ESKD) is considered a public health problem in both developed and emerging countries. This study aimed to assess the epidemiological profile of ESKD patients undergoing hemodialysis (HD) in Sidi Bel Abbes city.

Methods: This retrospective, observational and descriptive study was conducted from January 2015 to December 2018 involving 698 patients in Renal replacement therapy, including 673 patients undergoing HD.

Results: The prevalence of ESKD was $805.57 \mathrm{pmp}$. Regarding the epidemiological profile of HD patients, there was a slight predominance of males: $373(55.4 \%) v s$. Females: $300(44.6 \%)$; sex-ratio 1.24) (p < 0.001). The mean age was $57.43 \pm 17.05$ years. Patients included in age group 45-64 years were the most affected by ESKD (39.2\%) and hypertensive nephropathy and diabetic nephropathy were the most frequent causes (34.5\%) and (25.1\%) respectively, followed by unknown causes $(19.6 \%)$. Cardiovascular diseases were the major common comorbidities $(58.5 \%)$ ( $\mathrm{p}=0.043)$. The mean duration on HD was 5 years. 140 deaths $(20.8 \%)$ were registered, with males' predominance $61.4 \%$. The causes of death were primarily cardiovascular (41.4\%).

Conclusion: Our findings showed alarming rates in ESKD prevalence and incidence in Sidi Bel Abbes city, representing a burden for the country, affecting especially the young population. This study represents the first comprehensive description of clinical and epidemiological profile of HD treated ESKD patients in this city. This would contribute establishing an ESKD index dedicated to this city and creating a Maghreb or even an Arabic registry aiming to ensure better management of this pathology.
\end{abstract}

\section{Introduction}

Chronic Kidney Diseases (CKD) represent an enormous and increasing public health issue leading to a lifelong medical condition with profound impact on patients, families and healthcare providers. ${ }^{1}$ The number of patients undergoing renal replacement therapy (RRT) has exceeded 2.5 million and projected to reach 5.4 million in 2030. ${ }^{2}$ Based on the 2015 Global Burden of Disease Study from 1980 to 2015 , kidney disease was the 12 th most common cause of death. Overall CKD mortality has increased by $31.7 \%$ over the last 10 years, making it one of the fastest rising major causes of death, alongside diabetes and dementia. ${ }^{3}$ Thus, in 2017, CKD was listed as the 12th leading cause of death in the world, displaying an important elevation after being the 17th in $1990 .^{2}$ In Europe, CKD affects almost 94.1 million individuals, while 110 million cases are globally diagnosed in Africa. ${ }^{2}$

The clinical progression of this pathology to severe stage is frequently manifested by a progressive loss of nephron function, eventually leading to End Stage Kidney Diseases (ESKD), requiring treatment by RRT. ${ }^{4}$ It is obvious that the number of elderly patients suffering from diabetes, cardiovascular disease and hypertension is increasing and the poor outcomes of these endemic pathologies are mainly due to CKD that increases the global prevalence and incidence of $\mathrm{ESKD}^{5}$ becoming a major public health concern in both developed and emerging countries with varying prevalence. The effects of ESKD are strongly influenced by RRT, the cost of health care and the socio-economic level of the country concerned. ${ }^{5}$

\footnotetext{
* Corresponding author.

E-mail address: dzbengharez@yahoo.fr (Z. Bengharez).
} 
In Algeria, 3.5 million people were recorded with all-stage CKD. ${ }^{2}$ Furthermore, the number of patients suffering from ESKD was almost 25.000 cases with an estimated prevalence of $600 \mathrm{pmp}$ (patients per million population) and an incidence of $200 \mathrm{pmp}^{6}$

This current study represents the first comprehensive description of clinical and epidemiological profile of HD treated ESKD patients in Sidi Bel Abbes city (Western Algeria) using data collected from four dialysis centers. These data can help establishing an index of ESKD dedicated to the town of Sidi Bel Abbes with the purpose to ensure better management of this pathology.

\section{Materials and methods}

This retrospective, observational and descriptive study was carried out from January 2015 to December 2018 in four HD centers located in Sidi Bel Abbes city $\left(35^{\circ} 11^{\prime} 38^{\prime \prime} \mathrm{N} / 0^{\circ} 38^{\prime} 29^{\prime \prime} \mathrm{W}\right)$, Algeria, three from the private health care sector and one from the public sector (University Hospital). Only 15 year old patients and above with ESKD and treated by HD were included in the present study. However, patients with acute renal failure, transplantation failure during the period of our study and those who reside outside the area of Sidi Bel Abbes city were excluded.
All patients who had unusable or incomplete medical files were excluded too. Estimated Glomerular filtration rate (eGFR) was calculated using the abbreviated MDRD (Modification of Diet in Renal Disease) formula ${ }^{7}$ and the creatinine clearance was calculated using the Cockroft-Gault formula. ${ }^{8}$

\subsection{Statistical analysis}

Collected data were analyzed using IBM SPSS Statistics for Microsoft Windows version 23.0. Continuous variables were expressed as Mean \pm Standard deviation (SD) and categorical variables as percentages. The Student's t-test was used for group's comparisons. The one way analysis of variance (ANOVA) was performed for continuous variables, chisquare test and equivalents for categorical variables. The level of significance was set at $\mathrm{p}<0.05$.

\section{Results}

\subsection{Description of study population}

Since January 2015, a total of 698 patients were included in RRT,

Table 1

Demographic profile of patients overall, by gender and primary renal disease diagnosis.

\begin{tabular}{|c|c|c|c|c|c|c|c|c|c|c|c|c|c|c|}
\hline \multirow[t]{2}{*}{ Variables } & \multicolumn{2}{|l|}{ Gender } & \multirow[t]{2}{*}{$P$-value } & \multicolumn{9}{|c|}{ Primary renal disease diagnosis } & \multirow[t]{2}{*}{$P$-value } & \multirow[t]{2}{*}{ Overall } \\
\hline & $\begin{array}{l}\text { Male n } \\
(\%)\end{array}$ & $\begin{array}{l}\text { Female } \mathrm{n} \\
(\%)\end{array}$ & & HTN & DN & UKNC & GN & ADPKD & TLD & MLS & $\mathrm{VN}$ & OTU & & \\
\hline $\mathrm{N}(\%)$ & $\begin{array}{l}373 \\
(55.4)\end{array}$ & $\begin{array}{l}300 \\
(44.6)\end{array}$ & & $\begin{array}{l}232 \\
(34.5)\end{array}$ & $\begin{array}{l}169 \\
(25.1)\end{array}$ & $\begin{array}{l}132 \\
(19.6)\end{array}$ & $\begin{array}{l}51 \\
(7.6)\end{array}$ & $24(3.6)$ & $\begin{array}{l}21 \\
(3.1)\end{array}$ & $\begin{array}{l}17 \\
(2.5)\end{array}$ & $\begin{array}{l}14 \\
(2.1)\end{array}$ & $\begin{array}{l}13 \\
(1.9)\end{array}$ & & $\begin{array}{l}673 \\
(100)\end{array}$ \\
\hline Gender & / & / & / & & & & & & & & & & 0.338 & \\
\hline Male & / & / & & $\begin{array}{l}127 \\
(54.7)\end{array}$ & $\begin{array}{l}94 \\
(55.6)\end{array}$ & $66(50)$ & $\begin{array}{l}32 \\
(62.7)\end{array}$ & $\begin{array}{l}13 \\
(54.2)\end{array}$ & $\begin{array}{l}11 \\
(52.4)\end{array}$ & $\begin{array}{l}12 \\
(70.6)\end{array}$ & $7(50)$ & $\begin{array}{l}11 \\
(84.6)\end{array}$ & & $\begin{array}{l}373 \\
(55.4)\end{array}$ \\
\hline Female & / & / & & $\begin{array}{l}105 \\
(45.3)\end{array}$ & $\begin{array}{l}75 \\
(44.4)\end{array}$ & $66(50)$ & $\begin{array}{l}19 \\
(37.3)\end{array}$ & $\begin{array}{l}11 \\
(45.8)\end{array}$ & $\begin{array}{l}10 \\
(47.6)\end{array}$ & $\begin{array}{l}5 \\
(29.4)\end{array}$ & $7(50)$ & $\begin{array}{l}2 \\
(15.4)\end{array}$ & & $\begin{array}{l}300 \\
(44.6)\end{array}$ \\
\hline Age (years) & / & / & 0.705 & & & & & & & & & & $<0.001$ & \\
\hline 0-19 & $8(2.1)$ & $3(1)$ & & $1(0.4)$ & $0(0)$ & $2(1.5)$ & $2(3.9)$ & $0(0)$ & $1(4.8)$ & $\begin{array}{l}5 \\
(29.4)\end{array}$ & $0(0)$ & $0(0)$ & & 11(1.6) \\
\hline $20-44$ & $\begin{array}{l}80 \\
(21.4)\end{array}$ & $64(21.3)$ & & $\begin{array}{l}42 \\
(18.1)\end{array}$ & $27(16)$ & $37(28)$ & $\begin{array}{l}16 \\
(31.4)\end{array}$ & $4(16.7)$ & $\begin{array}{l}7 \\
(33.3)\end{array}$ & $\begin{array}{l}7 \\
(41.2)\end{array}$ & $1(7.1)$ & $\begin{array}{l}3 \\
(23.1)\end{array}$ & & $\begin{array}{l}144 \\
(21.4)\end{array}$ \\
\hline $45-64$ & $\begin{array}{l}145 \\
(38.9)\end{array}$ & $\begin{array}{l}110 \\
(36.7)\end{array}$ & & $\begin{array}{l}99 \\
(42.7)\end{array}$ & $\begin{array}{l}63 \\
(37.3)\end{array}$ & $\begin{array}{l}45 \\
(34.1)\end{array}$ & $\begin{array}{l}18 \\
(35.3)\end{array}$ & $\begin{array}{l}10 \\
(41.7)\end{array}$ & $\begin{array}{l}8 \\
(38.1)\end{array}$ & $\begin{array}{l}3 \\
(17.6)\end{array}$ & $\begin{array}{l}6 \\
(42.9)\end{array}$ & $\begin{array}{l}3 \\
(23.1)\end{array}$ & & $\begin{array}{l}255 \\
(37.9)\end{array}$ \\
\hline $65-74$ & $\begin{array}{l}78 \\
(20.9)\end{array}$ & $71(23.7)$ & & 44(19) & $\begin{array}{l}53 \\
(31.4)\end{array}$ & $\begin{array}{l}24 \\
(18.2)\end{array}$ & $\begin{array}{l}10 \\
(19.6)\end{array}$ & $4(16.7)$ & $\begin{array}{l}3 \\
(14.3)\end{array}$ & $\begin{array}{l}2 \\
(11.8)\end{array}$ & $\begin{array}{l}5 \\
(35.7)\end{array}$ & $\begin{array}{l}4 \\
(30.8)\end{array}$ & & $\begin{array}{l}149 \\
(22.1)\end{array}$ \\
\hline$\geq 75$ & $\begin{array}{l}62 \\
(16.6)\end{array}$ & $52(17.3)$ & & $\begin{array}{l}46 \\
(19.8)\end{array}$ & $\begin{array}{l}26 \\
(15.4)\end{array}$ & $\begin{array}{l}24 \\
(18.2)\end{array}$ & $5(9.8)$ & $6(25)$ & $2(9.5)$ & $0(0)$ & $\begin{array}{l}2 \\
(14.3)\end{array}$ & $\begin{array}{l}3 \\
(23.1)\end{array}$ & & $\begin{array}{l}114 \\
(16.9)\end{array}$ \\
\hline Marital status & & & $<0.001$ & & & & & & & & & & $<0.001$ & \\
\hline Single & $\begin{array}{l}87 \\
(23.3)\end{array}$ & $78(26)$ & & $\begin{array}{l}45 \\
(19.4)\end{array}$ & $22(13)$ & $\begin{array}{l}40 \\
(30.3)\end{array}$ & $\begin{array}{l}32 \\
(62.7)\end{array}$ & $7(29.2)$ & $\begin{array}{l}5 \\
(23.8)\end{array}$ & $\begin{array}{l}6 \\
(35.3)\end{array}$ & $\begin{array}{l}2 \\
(14.3)\end{array}$ & $\begin{array}{l}6 \\
(46.2)\end{array}$ & & $\begin{array}{l}165 \\
(24.5)\end{array}$ \\
\hline Married & $\begin{array}{l}264 \\
(70.8)\end{array}$ & 159(53) & & $\begin{array}{l}162 \\
(69.8)\end{array}$ & $\begin{array}{l}125 \\
(74)\end{array}$ & $\begin{array}{l}82 \\
(62.1)\end{array}$ & $\begin{array}{l}12 \\
(23.5)\end{array}$ & $\begin{array}{l}10 \\
(41.7)\end{array}$ & $\begin{array}{l}14 \\
(66.7)\end{array}$ & $\begin{array}{l}10 \\
(58.8)\end{array}$ & $\begin{array}{l}4 \\
(28.6)\end{array}$ & $\begin{array}{l}4 \\
(30.8)\end{array}$ & & $\begin{array}{l}423 \\
(62.9)\end{array}$ \\
\hline Divorced & $\begin{array}{l}12 \\
(3.2)\end{array}$ & $23(7.7)$ & & $8(3.4)$ & $6(3.6)$ & $6(4.5)$ & $4(7.8)$ & $5(20.8)$ & $2(9.5)$ & $1(5.9)$ & $1(7.1)$ & $\begin{array}{l}2 \\
(15.4)\end{array}$ & & $35(5.2)$ \\
\hline Widow & $\begin{array}{l}10 \\
(2.7)\end{array}$ & $40(13.3)$ & & $\begin{array}{l}17 \\
(7.3)\end{array}$ & $\begin{array}{l}16 \\
(9.5)\end{array}$ & $4(3)$ & $3(5.9)$ & $2(8.3)$ & $0(0)$ & $0(0)$ & $7(50)$ & $1(7.7)$ & & $50(7.4)$ \\
\hline $\begin{array}{l}\text { Socioprofessional } \\
\text { activities }\end{array}$ & & & 0.000 & & & & & & & & & & $<0.001$ & \\
\hline Unemployed & $\begin{array}{l}147 \\
(39.4)\end{array}$ & $126(42)$ & & $\begin{array}{l}104 \\
(44.8)\end{array}$ & $\begin{array}{l}52 \\
(30.8)\end{array}$ & $\begin{array}{l}55 \\
(41.7)\end{array}$ & $\begin{array}{l}19 \\
(37.3)\end{array}$ & $\begin{array}{l}15 \\
(62.5)\end{array}$ & $\begin{array}{l}9 \\
(42.9)\end{array}$ & $\begin{array}{l}9 \\
(52.9)\end{array}$ & $\begin{array}{l}5 \\
(35.7\end{array}$ & $\begin{array}{l}5 \\
(38.5)\end{array}$ & & $\begin{array}{l}273 \\
(40.6)\end{array}$ \\
\hline Housewives & $0(0)$ & $138(46)$ & & $\begin{array}{l}50 \\
(21.6)\end{array}$ & $\begin{array}{l}41 \\
(24.3)\end{array}$ & $\begin{array}{l}21 \\
(15.9)\end{array}$ & $\begin{array}{l}10 \\
(19.6)\end{array}$ & $6(25)$ & $\begin{array}{l}3 \\
(14.3)\end{array}$ & $1(5.9)$ & $\begin{array}{l}5 \\
(35.7)\end{array}$ & $1(7.7)$ & & $\begin{array}{l}138 \\
(20.5)\end{array}$ \\
\hline Retired workers & $\begin{array}{l}126 \\
(33.8)\end{array}$ & $22(7.3)$ & & $\begin{array}{l}40 \\
(17.2)\end{array}$ & $\begin{array}{l}43 \\
(25.4)\end{array}$ & $\begin{array}{l}38 \\
(28.8)\end{array}$ & $\begin{array}{l}10 \\
(19.6)\end{array}$ & $2(8.3)$ & $\begin{array}{l}5 \\
(23.8)\end{array}$ & $\begin{array}{l}2 \\
(11.8)\end{array}$ & $\begin{array}{l}2 \\
(14.3)\end{array}$ & $\begin{array}{l}6 \\
(46.2)\end{array}$ & & $\begin{array}{l}148 \\
(22)\end{array}$ \\
\hline $\begin{array}{l}\text { Long term sick leave } \\
\text { of absence }\end{array}$ & $\begin{array}{l}42 \\
(11.3)\end{array}$ & $6(2)$ & & $\begin{array}{l}20 \\
(8.6)\end{array}$ & $\begin{array}{l}17 \\
(10.1)\end{array}$ & $8(6.1)$ & $2(3.9)$ & $0(0)$ & $0(0)$ & $0(0)$ & $1(7.1)$ & $0(0)$ & & $48(7.1)$ \\
\hline $\begin{array}{l}\text { Independent } \\
\text { workers }\end{array}$ & $\begin{array}{l}33 \\
(8.8)\end{array}$ & $2(0.7)$ & & $14(6)$ & $\begin{array}{l}13 \\
(7.7)\end{array}$ & $1(0.8)$ & $3(5.9)$ & $1(4.2)$ & $1(4.8)$ & $1(5.9)$ & $0(0)$ & $1(7.7)$ & & $35(5.2)$ \\
\hline Full -time employed & $\begin{array}{l}14 \\
(3.8)\end{array}$ & $4(1.3)$ & & $2(0.9)$ & $2(1.2)$ & $6(4.5)$ & $4(7.8)$ & $0(0)$ & $\begin{array}{l}3 \\
(14.3)\end{array}$ & $0(0)$ & $1(7.1)$ & $0(0)$ & & $18(2.7)$ \\
\hline Students & $\begin{array}{l}11 \\
(2.9)\end{array}$ & $2(0.7)$ & & $2(0.9)$ & $1(0.6)$ & $3(2.3)$ & $3(5.9)$ & $0(0)$ & $0(0)$ & $\begin{array}{l}4 \\
(23.5)\end{array}$ & $0(0)$ & $0(0)$ & & $13(1.9)$ \\
\hline
\end{tabular}

Note: HTN: Hypertensive Nephropathy; DN: Diabetic Nephropathy; UKNC:Unknown Cause; GN: Chronic Glomerulonephritis; ADPKD: Autosomal Dominant Polycystic kidney Disease; TLD: Tubulointerstitial Disease; OTU: Obstructive Uropathy; VN:VascularNephropathy; MLS: Miscellaneous. 
treated in four dialysis centers in the Sidi Bel Abbes city. Eight-teen medical files were excluded due of incomplete data. A total of 673 patients (96\%) were treated by HD, 17 patients $(2.4 \%)$ by PD, and only 8 (1.1\%) with RT. 427 HD patients $(63.4 \%)$ were treated in the private sector treatment centers, while $246(36.6 \%)$ were treated in the public sector treatment center.

Prevalence rates of ESKD were estimated at $805.57 \mathrm{pmp}$ (863.7 pmp for males $v s .748 .31 \mathrm{pmp}$ for females). During the period 2015-2017, the incidence rates have been decreasing gradually from $164.89 \mathrm{pmp}$ to 140.56 pmp. However, in 2018 an increase has been recorded reaching 163.23 pmp.

\subsection{Profile of participants overall by gender}

The baseline characteristics of patients are summarized in Tables 1 and 2. There was a slight predominance of males: $373(55.4 \%)$ compared to females: 300 (44.6\%) with a sex-ratio 1.24. The mean age was $57.43 \pm 17.05$ years overall $(\mathrm{N}=673)(\mathrm{p}<0.001)$ while the median was 60 years. A peak was reported (39.2\%) in the 45-64 years age group representing the most affected cohort by ESKD.

In overall $62.9 \%(n=423)$ of patients were married. The distribution of marital and professional situation distribution was significantly different by gender $(\mathrm{p}<0.001)$.

Concerning etiologies, hypertensive nephropathy (HTN) being predominant (34.5\%) followed by diabetic nephropathy (DN) (25.1\%), both represented the most frequent etiologies regardless of gender, while unknown causes represented just over a quarter of the population studied.

Concerning cardiovascular risk, two factors were predominant: high blood pressure being diagnosed in $42.8 \%$ of patients, while diabetes was identified in $25.9 \%$. A positive history of smoking was detected in 13.5\%.Cardiovascular diseases were the most recurrent comorbidities covering $58.5 \%$ of our sample with 458 reported cases followed by respiratory pathologies $(14.9 \%)$. The distribution of comorbidities was significantly different between males and females $(p=0.043)$. Regarding serological status, $14.6 \%$ of the study population displayed a positive status for the hepatitis $\mathrm{C}$ virus (HCV) while hepatitis B (HBV) was found in $7.3 \%$. The distribution of HCV and HBV was identical in both genders as shown in Table 2. The HBV vaccination policy covered $44 \%$ of patients. No case of HIV infection was recorded during our study.

Anemia was prevalent in $90.7 \%$ (cutoff taken $11 \mathrm{~g} / \mathrm{dL}$ ) and $5.3 \%$ had hemoglobin levels below $7 \mathrm{~g} / \mathrm{dL}$ while values below $10 \mathrm{~g} / \mathrm{dL}$ were observed in $94.4 \%$ of our participants. The estimated average level of hemoglobin was $8.17 \pm 6.42 \mathrm{~g} / \mathrm{dL}$ with a significant difference by gender $(\mathrm{p}=0.005)$.

Furthermore, at the start of HD treatment, the estimated serum creatinine concentration was $105 \pm 39.56 \mathrm{mg} / \mathrm{L}$ varying within a range of $21-288 \mathrm{mg} / \mathrm{L}$. According to the Cockcroft and Gault formula, the mean creatinine clearance was $8.86 \pm 4.03 \mathrm{~mL} / \mathrm{min} / 1.73 \mathrm{~m}^{2}$ with limits ranging from 01 to $15 \mathrm{~mL} / \mathrm{min} / 1.73 \mathrm{~m}^{2}$. During the investigation period, $88.7 \%$ of patients had started their HD treatment with creatinine clearance $<10 \mathrm{~mL} / \mathrm{min} / 1.73 \mathrm{~m}^{2}$.

Among serum electrolytes, hyperkalemia was reported in $38.5 \%$ of patients while hypernatremia and hyponatremia were observed in $5.1 \%$ and $21 \%$ respectively. Moreover, hypocalcemia and hyperphosphatemia were screened in $51.7 \%$ and $60 \%$ respectively. All biological parameters were identical in men and women (Table 2).

The distribution across primary kidney disease was significant $(\mathrm{p}<$ 0.001). Patients with HTN or DN were older ( $p<0.001$ ), more likely to be male sex, to be married and unemployed (Table 1) and even more likely to be affected by cardiovascular diseases (Table 2). In contrast, patients with obstructive uropathy (OTU) were more likely to be female sex, single, retired or unemployed while patients with unknown cause were younger and peaked in the 45-64 age group, were married and unemployed reflecting an identical distribution among males and females while those with Glomerulonephritis (GN) were mostly older too,
Table 2

Clinical characteristics of HD patients overall and by gender.

\begin{tabular}{|c|c|c|c|c|}
\hline Variables & $\begin{array}{l}\text { Male } \mathrm{n} \\
(\%)\end{array}$ & $\begin{array}{l}\text { Female } \mathrm{n} \\
(\%)\end{array}$ & $\begin{array}{l}\text { Overall n } \\
(\%)\end{array}$ & $P$-value \\
\hline $\mathrm{N}(\%)$ & $\begin{array}{l}373 \\
(55.4)\end{array}$ & $\begin{array}{l}300 \\
(44.6)\end{array}$ & $673(100)$ & \\
\hline Background nephropathy & & & & 0.338 \\
\hline Hypertensive Nephropathy & $\begin{array}{l}127 \\
(34)\end{array}$ & $105(35)$ & $\begin{array}{l}232 \\
(34.5)\end{array}$ & \\
\hline Diabetic Nephropathy & $\begin{array}{l}94 \\
(25.2)\end{array}$ & $75(25)$ & $\begin{array}{l}169 \\
(25.1)\end{array}$ & \\
\hline Unknown cause & $\begin{array}{l}66 \\
(17.7)\end{array}$ & $66(22)$ & $\begin{array}{l}132 \\
(19.6)\end{array}$ & \\
\hline Chronic Glomerulonephritis & $32(8.6)$ & $19(6.3)$ & $51(7.6)$ & \\
\hline ADPKD & $13(3.5)$ & 11(3.7) & $24(3.6)$ & \\
\hline Tubulointerstitial Disease & $11(2.9)$ & $10(3.3)$ & $21(3.1)$ & \\
\hline Miscellaneous & $12(3.2)$ & $5(1.7)$ & $17(2.5)$ & \\
\hline Vascular Nephropathy & $7(1.9)$ & $7(2.3)$ & $14(2.1)$ & \\
\hline Obstructive Uropathy & $11(2.9)$ & $2(0.7)$ & 13(1.9) & \\
\hline Comorbidities & & & & 0.043 \\
\hline Cardiovascular diseases & $\begin{array}{l}261 \\
(70)\end{array}$ & $\begin{array}{l}133 \\
(44.3)\end{array}$ & $\begin{array}{l}394 \\
(58.5)\end{array}$ & \\
\hline Respiratory pathologies & $28(7.5)$ & $72(24)$ & $\begin{array}{l}100 \\
(14.9)\end{array}$ & \\
\hline Digestive diseases & $31(8.3)$ & $23(7.7)$ & $54(8)$ & \\
\hline Otorhinolaryngology & $19(5.1)$ & $15(5)$ & $34(5.1)$ & \\
\hline Endocrine diseases & $7(1.9)$ & $20(6.7)$ & $27(4)$ & \\
\hline Miscellaneous & $27(7.2)$ & $37(12.3)$ & $64(9.5)$ & \\
\hline Smoking & $\begin{array}{l}89 \\
(23.9)\end{array}$ & $2(0.7)$ & $91(13.5)$ & $<0.001$ \\
\hline HCV positive & $\begin{array}{l}58 \\
(15,5)\end{array}$ & $40(13,3)$ & $98(14.6)$ & 0.560 \\
\hline HBV positive & $27(7.2)$ & $22(7.3)$ & $49(7.3)$ & 0.788 \\
\hline HCV\& HBV positive & $11(2.9)$ & $5(1.7)$ & $16(2.4)$ & 0.421 \\
\hline $\mathrm{Hb}$ levels $(\mathrm{g} / \mathrm{dl})$ & & & & 0.005 \\
\hline$>11$ & $\begin{array}{l}45 \\
(12.1)\end{array}$ & $17(5.7)$ & $62(9.3)$ & \\
\hline 7-11 & $\begin{array}{l}302 \\
(81)\end{array}$ & 273(91) & $\begin{array}{l}575 \\
(85.4)\end{array}$ & \\
\hline$<7$ & $26(7)$ & $10(3.3)$ & $36(5.3)$ & \\
\hline $\begin{array}{l}\text { Clearance of serum creatinine } \\
\text { levels }\left(\mathrm{ml} / \mathrm{min} / 1.73 \mathrm{~m}^{2}\right)\end{array}$ & & & & 0.062 \\
\hline $6-9$ & $\begin{array}{l}153 \\
(41)\end{array}$ & $\begin{array}{l}136 \\
(45.3)\end{array}$ & $\begin{array}{l}289 \\
(42.9)\end{array}$ & \\
\hline $9.5-11$ & $\begin{array}{l}69 \\
(18.5)\end{array}$ & $62(20.7)$ & $\begin{array}{l}131 \\
(19.5)\end{array}$ & \\
\hline $3.5-5.5$ & $\begin{array}{l}77 \\
(20.6)\end{array}$ & $44(14.7)$ & $121(18)$ & \\
\hline$\leq 3$ & $34(9.1)$ & $31(10.3)$ & $65(9.7)$ & \\
\hline $11.50-13$ & $22(5.9)$ & $15(5)$ & $37(5.5)$ & \\
\hline $13.50-15$ & $18(4.8)$ & $12(4)$ & $30(4.5)$ & \\
\hline Serum Potassium levels (mEq/L) & & & & 0.771 \\
\hline$<3.5$ & $15(4)$ & $7(2.3)$ & $22(3.2)$ & \\
\hline $3.5-5.5$ & $\begin{array}{l}213 \\
(57.1)\end{array}$ & 179(597) & $\begin{array}{l}392 \\
(58.3)\end{array}$ & \\
\hline$>5.5$ & $\begin{array}{l}145 \\
(38.9)\end{array}$ & $114(38)$ & $\begin{array}{l}259 \\
(38.5)\end{array}$ & \\
\hline Serum Sodium levels (mEq/L) & & & & 0.252 \\
\hline$<135$ & $\begin{array}{l}81 \\
(21.7)\end{array}$ & $60(20)$ & $141(21)$ & \\
\hline 135-155 & $\begin{array}{l}274 \\
(73.5)\end{array}$ & $\begin{array}{l}224 \\
(74.7)\end{array}$ & $498(74)$ & \\
\hline$>155$ & $18(4.8)$ & $16(5.3)$ & $34(5.1)$ & \\
\hline Serum Calcium levels (mg/dl) & & & & 0,532 \\
\hline$<8,5$ & $\begin{array}{l}179 \\
(48)\end{array}$ & $\begin{array}{l}169 \\
(56.3)\end{array}$ & $\begin{array}{l}348 \\
(51.7)\end{array}$ & \\
\hline $8,5-10,5$ & $\begin{array}{l}172 \\
(46.1)\end{array}$ & $153(41)$ & $\begin{array}{l}325 \\
(48.3)\end{array}$ & \\
\hline$>10,5$ & $0(0)$ & $0(0)$ & $0(0)$ & \\
\hline Serum Phosphorus levels (mg/dl) & & & & 0,443 \\
\hline$<2.5$ & $0(0)$ & $0(0)$ & $0(0)$ & \\
\hline $2.5-4.5$ & $\begin{array}{l}138 \\
(37)\end{array}$ & $\begin{array}{l}131 \\
(43.7)\end{array}$ & $269(40)$ & \\
\hline$>4.5$ & $\begin{array}{l}209 \\
(56)\end{array}$ & 195(65) & $404(60)$ & \\
\hline
\end{tabular}

Note: Hb: Hemoglobin;ADPKD:Autosomal Dominant Polycystic Kidney Disease. 
more likely to be males, to be single and unemployed (Table 1). Moreover, respiratory pathologies were most common risks factors in $46.2 \%$. In general comorbidities' distribution across background nephropathy was statistically significant $(\mathrm{p}<0.001$ ). Significant differences were also recorded in all variations of laboratory parameters among background nephropathies as shown in Table 3.

Most of patients (97\%) received dialysis three times per week for an average cumulative weekly dose of $683 \mathrm{~min}$, with an average of $720 \mathrm{~min}$ (12 h per week).

Our results showed that $78 \%$ of investigated patients were not undergoing nephrological follow-up prior starting HD; however only $22 \%$ were followed by a nephrologist before starting their HD treatment. The majority of diagnosed patients (98.5\%) were treated first by HD for an estimated average period of $3 \pm 0.58$ months further the diagnosis. The first HD session was often performed using a central venous catheter in $91 \%$ of cases. $65.5 \%$ of patients had their fistulas placed over three months and after the commencement of HD treatment while $12 \%$ had the fistulas further six months. Renal biopsy accounted for a small proportion (4\%).

On the other hand, $82.3 \%$ of patients were independent in their daily lives and capable of walking on their own, $17.7 \%$ were not and $15.7 \%$ needed a third person while $2 \%$ were totally physically disabled.

In general, the mean duration on HD was 5 years (Fig. 1) estimated at $3.5 \pm 1.32$ years for patients with HTN and $2.9 \pm 0.43$ years for those with DN with a confidence interval CI $95 \%=[2.47-3.43]$ years.

It should be noticed that 140 deaths $(20.8 \%)$ were registered during

Table 3

Clinical characteristics of patients by primary renal disease diagnosis.

\begin{tabular}{|c|c|c|c|c|c|c|c|c|c|c|}
\hline Variables & HTN & DN & UKNC & GN & ADPKD & TLD & MLS & $\mathrm{VN}$ & OTU & $P$-value \\
\hline $\mathrm{N}(\%)$ & $\begin{array}{l}232 \\
(34.5)\end{array}$ & $\begin{array}{l}169 \\
(25.1)\end{array}$ & $\begin{array}{l}132 \\
(19.6)\end{array}$ & $51(7.6)$ & $24(3.6)$ & $21(3.1)$ & $17(2.5)$ & $14(2.1)$ & 13(1.9) & \\
\hline Comorbidities & & & & & & & & & & $<0.001$ \\
\hline Cardiovascular diseases & $\begin{array}{l}185 \\
(79.7)\end{array}$ & $\begin{array}{l}105 \\
(62.1)\end{array}$ & $39(29.5)$ & $\begin{array}{l}31 \\
(60.8)\end{array}$ & $\begin{array}{l}16 \\
(66.7)\end{array}$ & $6(28.6)$ & $1(5.9)$ & $\begin{array}{l}10 \\
(71.4)\end{array}$ & $1(7.7)$ & \\
\hline Respiratory pathologies & $15(6.5)$ & $23(13.6)$ & $35(26.5)$ & $8(15.7)$ & $4(16.7)$ & $0(0)$ & $7(41.2)$ & $2(14.3)$ & $6(46.2)$ & \\
\hline Digestive diseases & $8(3.4)$ & $4(2.4)$ & $24(18.2)$ & $3(5.9)$ & $0(0)$ & $\begin{array}{l}11 \\
(52.4)\end{array}$ & $4(23.5)$ & $0(0)$ & $0(0)$ & \\
\hline Otorhinolaryngology & $5(2.2)$ & $7(4.1)$ & $10(7.6)$ & $2(3.9)$ & $1(4.2)$ & $1(4.8)$ & $3(17.6)$ & $1(7.1)$ & $4(30.8)$ & \\
\hline Endocrine diseases & $5(2.2)$ & $6(3.6)$ & $4(3)$ & $3(5.9)$ & $2(8.3)$ & $2(9.5)$ & $2(11.8)$ & $1(7.1)$ & $2(15.4)$ & \\
\hline Miscellaneous & $14(6)$ & $24(14.2)$ & $20(15.2)$ & $4(7.8)$ & $1(4.2)$ & $1(4.8)$ & $0(0)$ & $0(0)$ & $0(0)$ & \\
\hline Smoking & $26(11.2)$ & $21(12.4)$ & $13(9.8)$ & $\begin{array}{l}11 \\
(21.6)\end{array}$ & $6(25)$ & $6(28.6)$ & $2(11.8)$ & $2(14.3)$ & $4(30.8)$ & 0.474 \\
\hline HCV positive & $32(13.8)$ & 18(10.7) & $28(21.2)$ & $6(11.8)$ & $2(8.3)$ & $5(23.8)$ & $2(11.8)$ & $2(14.3)$ & $3(23.1)$ & 0.182 \\
\hline HBV positive & $14(6)$ & $12(7.1)$ & $14(10.6)$ & $5(9.8)$ & $1(4.2)$ & $1(4.8)$ & $1(5.9)$ & $0(0)$ & $1(7.7)$ & 0.389 \\
\hline HCV \& HBV positive & $6(2.6)$ & $5(3)$ & $3(2.3)$ & $0(0)$ & $0(0)$ & $0(0)$ & $2(11.8)$ & $0(0)$ & $0(0)$ & 0.489 \\
\hline Hb levels $(\mathrm{g} / \mathrm{dl})$ & & & & & & & & & & $<0.001$ \\
\hline$>11$ & $21(9.1)$ & $7(4.1)$ & $7(5.3)$ & $\begin{array}{l}10 \\
(19.6)\end{array}$ & $5(20.8)$ & $4(19)$ & $4(23.5)$ & $3(21.4)$ & $1(7.7)$ & \\
\hline 7-11 & $\begin{array}{l}198 \\
(85.3)\end{array}$ & $\begin{array}{l}158 \\
(93.5)\end{array}$ & $\begin{array}{l}122 \\
(92.4)\end{array}$ & $\begin{array}{l}37 \\
(72.5)\end{array}$ & $18(75)$ & $\begin{array}{l}15 \\
(71.4)\end{array}$ & $\begin{array}{l}10 \\
(58.8)\end{array}$ & $8(57.1)$ & $9(69.2)$ & \\
\hline$<7$ & $13(5.6)$ & $4(2.4)$ & $3(2.3)$ & $34(7.8)$ & $1(4.2)$ & $2(9.5)$ & $3(17.6)$ & $3(21.4)$ & $3(23.1)$ & \\
\hline $\begin{array}{l}\text { Clearance of serum creatinine levels }(\mathrm{ml} / \mathrm{min} / \\
\left.1.73 \mathrm{~m}^{2}\right)\end{array}$ & & & & & & & & & & 0.003 \\
\hline $6-9$ & $88(37.9)$ & $70(41.4)$ & $68(51.5)$ & $\begin{array}{l}20 \\
(39.2)\end{array}$ & $12(50)$ & $\begin{array}{l}10 \\
(47.6)\end{array}$ & $9(52.9)$ & $7(50)$ & $5(38.5)$ & \\
\hline $9.5-11$ & $55(23.7)$ & $39(23.1)$ & $22(16.7)$ & $5(9.8)$ & $3(12.5)$ & $3(14.3)$ & $2(11.8)$ & $1(7.1)$ & $1(7.7)$ & \\
\hline $3.5-5.5$ & $47(20.3)$ & $35(20.7)$ & $20(15.2)$ & $7(13.7)$ & $4(16.7)$ & $3(14.3)$ & $3(17.6)$ & $1(7.1)$ & $1(7.7)$ & \\
\hline$\leq 3$ & $20(8.6)$ & $14(8.3)$ & $12(9.1)$ & $6(11.8)$ & $5(20.8)$ & $4(19)$ & $3(17.6)$ & $1(7.1)$ & $0(0)$ & \\
\hline $11.50-13$ & $13(5.6)$ & $5(3)$ & $6(4.5)$ & $7(13.7)$ & $0(0)$ & $1(4.8)$ & $0(0)$ & $2(14.3)$ & $3(23.1)$ & \\
\hline $13.50-15$ & $9(3.9)$ & $6(3.6)$ & $4(3)$ & $6(11.8)$ & $0(0)$ & $0(0)$ & $0(0)$ & $2(14.3)$ & $3(23.1)$ & \\
\hline Serum Potassium levels (mEq/L) & & & & & & & & & & 0.036 \\
\hline$<3.5$ & $7(3)$ & $4(2.4)$ & $3(2.3)$ & $2(3.9)$ & $1(4.2)$ & $2(9.5)$ & $1(5.9)$ & $2(14.3)$ & $0(0)$ & \\
\hline $3.5-5.5$ & $\begin{array}{l}120 \\
(51.7)\end{array}$ & $99(58.6)$ & $76(57.6)$ & $\begin{array}{l}33 \\
(64.7)\end{array}$ & $\begin{array}{l}16 \\
(66.7)\end{array}$ & $\begin{array}{l}15 \\
(71.4)\end{array}$ & $\begin{array}{l}14 \\
(82.4)\end{array}$ & $9(64.3)$ & $\begin{array}{l}10 \\
(76.9)\end{array}$ & \\
\hline$>5.5$ & $\begin{array}{l}105 \\
(45.3)\end{array}$ & $66(39.1)$ & $53(40.2)$ & $\begin{array}{l}16 \\
(31.4)\end{array}$ & $7(29.2)$ & $4(19)$ & $2(11.8)$ & $3(21.4)$ & $3(23.1)$ & \\
\hline Serum Sodium levels (mEq/L) & & & & & & & & & & 0.022 \\
\hline$<135$ & $30(12.9)$ & $36(21.3)$ & $34(25.8)$ & $\begin{array}{l}16 \\
(31.4)\end{array}$ & $6(25)$ & $7(33.3)$ & $5(29.4)$ & $5(35.7)$ & $2(15.4)$ & \\
\hline 135-155 & $\begin{array}{l}197 \\
(84.9)\end{array}$ & $\begin{array}{l}122 \\
(72.2)\end{array}$ & $95(72)$ & $\begin{array}{l}31 \\
(60.8)\end{array}$ & $\begin{array}{l}17 \\
(70.8)\end{array}$ & $\begin{array}{l}11 \\
(52.4)\end{array}$ & $\begin{array}{l}10 \\
(58.8)\end{array}$ & $6(42.9)$ & $9(69.2)$ & \\
\hline$>155$ & $5(2.2)$ & $11(6.5)$ & $3(2.3)$ & $4(7.8)$ & $1(4.2)$ & $3(14.3)$ & $2(11.8)$ & $3(21.4)$ & $2(15.4)$ & \\
\hline Serum Calcium levels (mg/dl) & & & & & & & & & & 0,012 \\
\hline$<8,5$ & $\begin{array}{l}145 \\
(62.5)\end{array}$ & $90(38.8)$ & $74(31.9)$ & $5(2.2)$ & $2(0.9)$ & $18(7.8)$ & $7(3)$ & $4(1.7)$ & $3(1.3)$ & \\
\hline $8,5-10,5$ & $87(37.5)$ & $79(46.7)$ & $58(43.9)$ & $\begin{array}{l}46 \\
(90.2)\end{array}$ & $\begin{array}{l}22 \\
(91.7)\end{array}$ & $3(14.3)$ & $\begin{array}{l}10 \\
(58.8)\end{array}$ & $\begin{array}{l}10 \\
(71.4)\end{array}$ & $\begin{array}{l}10 \\
(76.9)\end{array}$ & \\
\hline$>10,5$ & $0(0)$ & $0(0)$ & $0(0)$ & $0(0)$ & $0(0)$ & $0(0)$ & $0(0)$ & $0(0)$ & $0(0)$ & \\
\hline Serum Phosphorus levels (mg/dl) & & & & & & & & & & 0.016 \\
\hline$<2.5$ & $0(0)$ & $0(0)$ & $0(0)$ & $0(0)$ & $0(0)$ & $0(0)$ & $0(0)$ & $0(0)$ & $0(0)$ & \\
\hline $2.5-4.5$ & $\begin{array}{l}113 \\
(48.7)\end{array}$ & $80(47.3)$ & $40(30.3)$ & $\begin{array}{l}19 \\
(37.3)\end{array}$ & $8(33.3)$ & $4(19)$ & $2(11.8)$ & $3(21.4)$ & $0(0)$ & \\
\hline$>4.5$ & $\begin{array}{l}119 \\
(51.3)\end{array}$ & $89(52.7)$ & $92(69.7)$ & $\begin{array}{l}32 \\
(62.7)\end{array}$ & $\begin{array}{l}16 \\
(66.7)\end{array}$ & $17(81)$ & $\begin{array}{l}15 \\
(88.2)\end{array}$ & $\begin{array}{l}11 \\
(78.6)\end{array}$ & $13(100)$ & \\
\hline
\end{tabular}

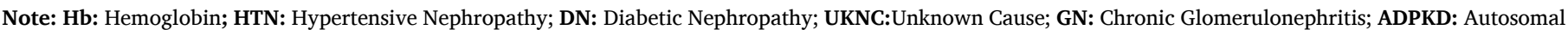
Dominant Polycystic kidney Disease; TLD: Tubulointerstitial Disease; OTU: Obstructive Uropathy; VN:Vascular Nephropathy; MLS: Miscellaneous. 


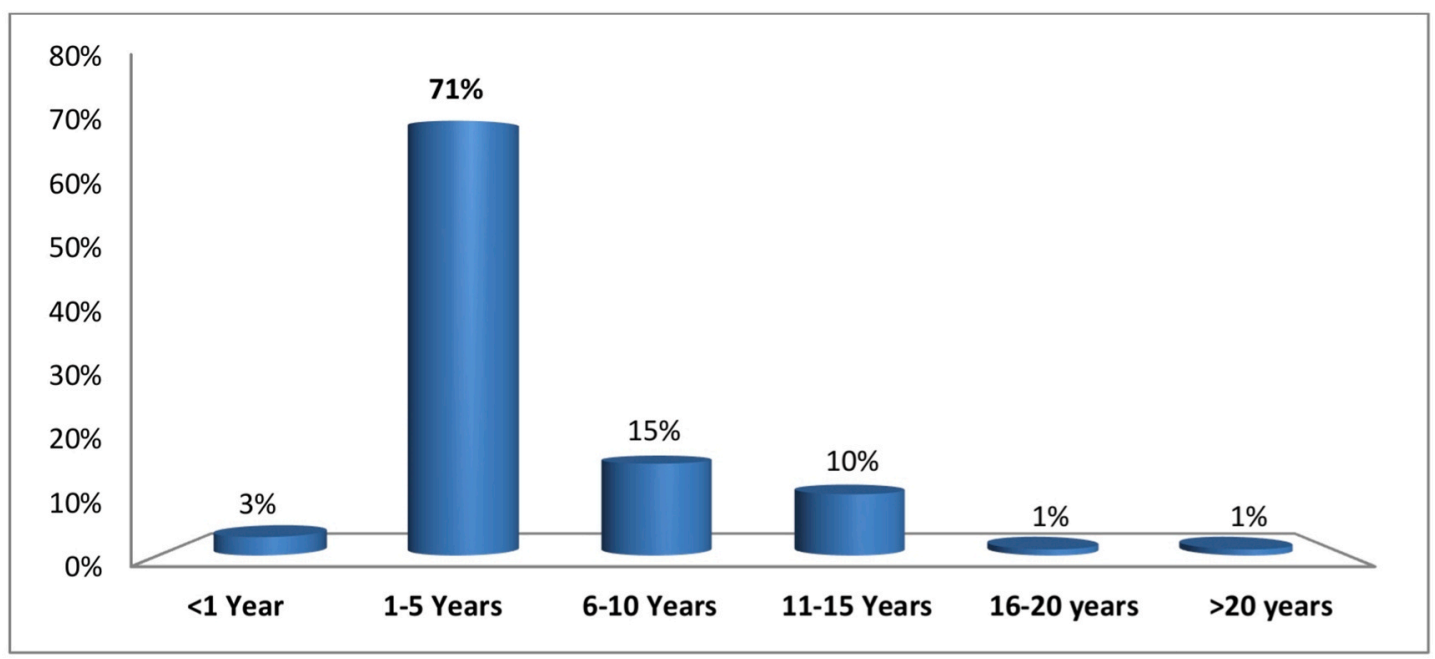

Fig. 1. Distribution according to duration on HD.

our survey, $61.4 \%$ were males and $38.6 \%$ were females $(\mathrm{p}=0.533)$.As illustrated on Fig. 2, the mortality rate tended to be elevated in males mainly due to cardiovascular causes $(41.4 \%)$, followed by metabolic causes (16\%). In HD, $33.6 \%$ of reported death cases were with DN where the most frequent causes of death were cardiovascular (8.9\%). Concerning HD patients with HTN, $32.9 \%$ of death cases were recorded, the most frequent causes were cardiovascular diseases (10.3\%) followed by cerebrovascular accident (5.2\%). The mean age of death was 65 years where the elderly patients represented $48 \%$ of the total deaths with an average duration on HD of $3 \pm 1.72$ years, with confidence interval $\mathrm{CI}_{95 \%}[2.21-3.79]$ years.

\section{Discussion}

The current study reflects the first comprehensive description of clinical and epidemiological profile of HD treated ESKD patients in Sidi Bel Abbes city (Western Algeria).The survey, providing some information on the profile of ESKD patients undergoing HD pointed out a worrying increase of prevalence and incidence of ESKD. The incidence rates we obtained are higher than those reported in other Algerian region $^{9}$ and similar to those observed from study in India (150-200 pmp). ${ }^{5}$
From our results, it was found that ESKD prevalence was higher than that reported from an Iranian study in 2017: $680 \mathrm{pmp}^{10}$ but lower compared to that of a French population study in 2016: (prevalence: 1262 pmp; incidence: 165 pmp). ${ }^{11}$ In 2009, some countries were known for their high prevalence rates such as the United States (USA): 1811 pmp, Japan: 2205 pmp $^{12}$, and Canada: 1118.7 pmp. ${ }^{13}$ HD was the predominant modality of RRT (96\%), this partly explains the high cost of this disease for our country, requiring a permanent mobilization of well trained and qualified medical, paramedical and technical healthcare staff. $^{9}$

RT represents only $1.1 \%$ because of the difficulties of access to this therapy and the refusal of certain Muslim families for religious reasons. However, it remains the best adapted treatment and the least expensive in the medium and long term for the patient, society and the paying agency. In our study, the private sector treatment centers play a crucial role in the treatment of ESKD (63.4\%).

ESKD affected both sexes with a slight predominance of male sex. These findings agree with some studies. ${ }^{13,14}$ The prevalence of ESKD varied considerably with age and remains more expressed in the young population as previously described in some Algerian investigations ${ }^{9,15}$ and even abroad. ${ }^{16}$ On the other hand, some studies showed a reduced

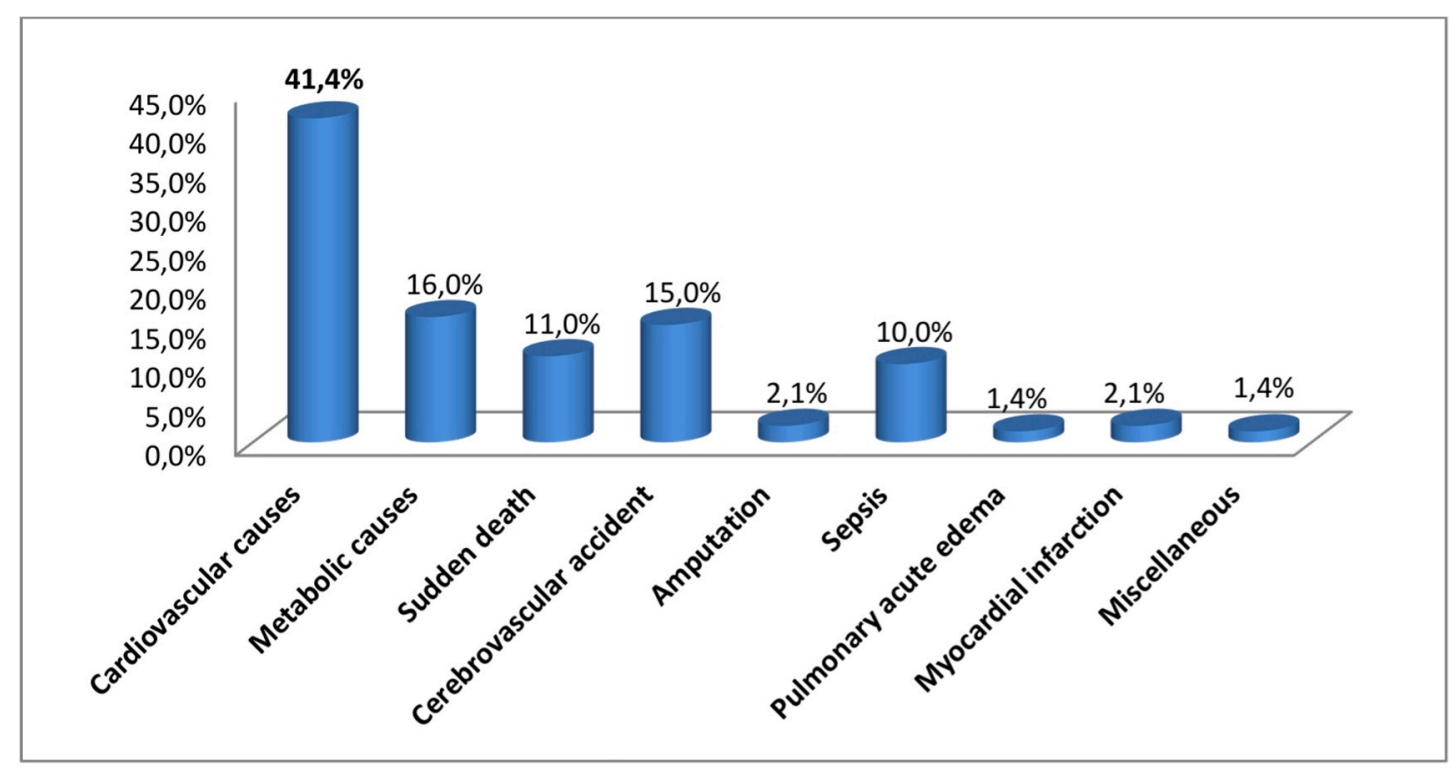

Fig. 2. Distribution of causes of death of HD patients. 
prevalence in elderly patients. ${ }^{11,17}$ It should be reminded that the high prevalence reported among the young population could be due to the significant difference in the distribution of background nephropathies in our patients highlighting the condition of this category of patients in some etiologies such as HTN, DN and unknown cause. Unemployment status was predominant in more than half of the patients including women especially housewives and young patients, which agrees with Yu-Yin Kao et al. results. ${ }^{18}$

The overall review of etiologies, related to ESKD of our patients, throughout the study period revealed such as several other studies that HTN and DN were the most common etiologies. These etiologies are mostly frequent among elderly patients representing the leading and major causes of ESKD. ${ }^{5,19}$ Similar trends were observed in study conducted at the United Arab Emirates (UAE), Dubai. ${ }^{17}$ GN was present at a reduced rate, this result is consistent with that of Bouhabel et al. ${ }^{9}$ The prevalence rate of unknown etiologies remains however significant, due to the late diagnosis of this disease and the low proportion of patients who can undertake renal biopsy(4\%). This can lead to a delayed referral of patients to HD and consequently the diagnosis of the initial cause becomes difficult to establish. The rate of undetermined causes recorded in our study is comparable to that found in certain local studies such as that of Bouhabel et al. $(26 \%)^{9}$ and Houat $(46 \%)^{20}$ and in foreign countries such as the UAE-Dubai: $10.95 \%{ }^{17}$ and France: $15.8 \%{ }^{11}$

In the current study, the majority of HD patients had at least one comorbidity.Cardiovascular diseases were the most frequent among our patients $(58.5 \%)$. Hence, it is becoming increasingly paramount to collaborate with cardiologists. Our findings highlights the relationship between ESKD comorbidities and cardiovascular complications. The predominance of metabolic pathologies, such as diabetes and hypertension, smoking, sedentary lifestyle and the severity of anemia at when starting the treatment represent the predisposing factors to this morbidity. Identical results were reported in the annual report of the French renal registry. ${ }^{11}$

Data on the hepatitis epidemiology have shown significant rates in our population, the proportions of HCV and HBV are comparable to those reported in others countries. ${ }^{17,21-23}$ Viral infections, especially those caused by HCV and HBV are frequent in patients undergoing HD, hence, HCV remains the principal viral infection in this population. The severity of this infection lies in its high risk, leading to the progression to chronicity and the development of cirrhosis or hepatocellular carcinoma. ${ }^{23,24}$ Duration on HD and blood transfusions remain major factors in the increase of hepatitis, infection control, disinfection of HD machines after performing HD sessions and isolation of patients with positive serology tested may reduce the risk of transmission of the infections.

In the present study, a high prevalence of anemia was detected (90.7\%), being a first major comorbidity of $\mathrm{ESKD}^{22}$ and the most important risk factor for left ventricular hypertrophy. This condition worsens with the progression of CKD and complicates ESKD. However, the treatment of anemia can have a significant impact on mortality, hospitalization, quality of life, brain and cognitive function, and exercise capacity among patients with ESKD. ${ }^{24}$ In view of these results, improving the management of anemia becomes necessary.

Our results for serum creatinine and serum creatinine clearance are consistent with findings from Moroccan studies ${ }^{21}$, in contrast, a study from Iran showed different levels with serum creatinine $>10 \mathrm{mg} / \mathrm{dl}$ and creatinine clearance $<10 \mathrm{ml} / \mathrm{min}$ in 40,3 and $61,3 \%$ of the patients, respectively. ${ }^{25}$ The cumulative delay in the rapid management of ESKD recorded in our study population could be the result of the high value of serum creatinine and creatinine clearance upon first admission to HD treatment.

Regarding electrolyte disturbances, a high percentage of emergency dialysis on a temporary catheter was observed, especially due to hyperkalemia or acute pulmonary edema. This further contributes to increasing the cost of this therapy and can be the cause of death, underlining the importance of the early diagnosis of this serious complication in order to ensure its management. Similar trends were reported by Korgaonkar et al. ${ }^{26}$ when studying relationship between serum potassium and mortality in CKD. Moreover, hyponatremia was detected in our patients and which can generally be corrected by fluid restriction, while the presence of hypernatremia may be due to the excessive salt intake. This can cause water retention; therefore, patients should regularly monitor their sodium intake. Hypocalcemia remains a frequent complication among patients with CKD requiring discontinuation of calcitriol or another vitamin D derivative. Thus, abnormal serum electrolytes are generally common among HD patients who should therefore be monitored regularly and treated with appropriate correction to avoid any worsening.

According to our data, the majority of patients underwent dialysis three times a week, this is in accordance with international recommendations. ${ }^{27}$ The lengthening of the time between the diagnosis of this pathology and the onset of HD can lead to complications in the management of ESKD.

The mean duration on HD was 5 years. This survival report is comparable to that of international centers, and similar to that of Qatar ${ }^{28}$ and the UAE ${ }^{17}$ However, RT remains the best RRT in terms of quality of life and survival rate of patients. ${ }^{10}$

Based on the obtained mortality rate of $20.8 \%$, our results are superior to those of France $(10.6 \%) .{ }^{11}$ It should be remembered that mortality rate represents a significant and indirect quality of health care indicator. In fact, this parameter depends on several factors such as the age of patients when initiating HD, background nephropathies, especially the presence of diabetes, and other comorbidities.

\section{Conclusion}

The results of our study showed a worrying increase of prevalence and incidence rates of ESKD patients in Sidi Bel Abbes city, making this pathology a major public health concern and a heavy burden for Algerian society, due to the rising number of patients with ESKD, especially the youngest ones, to the epidemiological transition of the country and to the high cost of its management.

The annual incidences recorded during our study indicate the etiologies providing ESKD (diabetes, hypertension) that require an improvement in the management of the general population in the future. This study could be helpful control the flow of ESKD with possessing reliable statistics. The establishment of centers dedicated to patients in a severe and complicated stage, especially the elderly and those suffering from multiple pathologies requiring the permanent support of a nephrologist is highly recommended. The founding of a Maghreb register, why not an Arabic register of ESKD becomes a necessity because of the rapid increase in the number of patients during the latest years.

As any study carried out on humans, the current study is not without its limitations. Certain difficulties were noted during data collection, due to the lack of information in patient's medical files. In addition, the establishment of an infrared network containing the digital medical record of each patient is strongly recommended, especially at the university hospital of Sidi Bel Abbes city.

\section{Ethical approval}

This study, a part of phD thesis of the first author, was approved by the Institutional Scientific Review Committee. All procedures contributing to this work comply with the ethical standards.

\section{Declaration of competing interest}

Authors declare having no conflict of interest regarding this study.

\section{Acknowledgements}

The authors would like to thank the Directorate General of Scientific 
Research and Technological Development (DGRSDT) and the Thematic Agency of Research in Biotechnology and Agri-Food Sciences (ATRBSA), Algeria, for their research support. Also, We are very thankful to the staff of Nephrology Department of the University Hospital of Sidi Bel Abbes city and the three private hemodialysis centers who facilitated and contributed to the successful of this study.

\section{References}

1 Wiam Abdulaziz A. Epidemiology of Dialysis-Treated End-Stage Kidney Disease in Adults in Libya. PhD thesis. University of Nottingham; 2013.

2 GBD Chronic Kidney Disease Collaboration. Global, regional, and national burden of chronic kidney disease, 1990-2017: a systematic analysis for the Global Burden of Disease Study 2017. Lancet. 2020;395(10225):709-733.

3 GBD 2015. Mortality and Causes of Death Collaborators. Global, regional, and national life expectancy, all-cause mortality, and cause-specific mortality for 249 causes of death. 1980-2015: a systematic analysis for the Global Burden of Disease Study 2015 [published correction appears in Lancet. 2017 Jan 7;389(10064):e1]. Lancet. 2016;388:1459-1544, 10053.

4 Sathyan S, George S, Vijayan P, Jayakumar M. Clinical and epidemiological profile of chronic kidney disease patients in a tertiary care referral centre in South India. Int J Community Med Public Health. 2016;3:3487-3492.

5 Lazars ER. Effectiveness of education and exercise on quality of life among patients undergoing hemodialysis. Clin Epidemiol Global Health. 2019;7:402-408.

6 Mime MI, Bellebia S, Bengharez Z, Louni M, Benrachedi K. Assessment and monitoring the quality oftreated water and dialysate in a public hemodialysis center to prevent potential health risks. Algerian J. Env. Sc. Technology. 2019;5(1):912-919.

7 Mani MK. Prevention of chronic renal failure at the community level. Kidney Int Suppl. 2003;(83):S86-S89.

8 Cockcroft DW, Gault MH. Prediction of creatinine clearance from serum creatinine. Nephron. 1976;16(1):31-41.

9 Bouhabel A, Laib Z, Hannache K, Aberkane A. Traitement de suppléance rénale par hémodialyse à Constantine (Algérie): état des lieux [Renal replacement therapy by hemodialysis in Constantine (Algeria)]. Néphrol Thérapeutique. 2014 Feb;10(1): $39-43$.

10 Moradpour A, Hadian M, Tavakkoli M. Economic evaluation of end stage renal disease treatments in Iran. Clin Epidemiol Glob Health. 2020;8:199-204.

11 de Biomédecine Agence. Rapport annuel. Réseau Epidémiologie et Information en Néphrologie (REIN). Registre français des traitements de suppléance de l'insuffisance rénale chronique; 2018. Retrieved from : https://www.francerein.org/actualites/2 3-les-donnees-du-rapport-rein.
12 Collins AJ, Foley RN, Chavers B, et al. 'United States renal data system 2011 annual data report: atlas of chronic kidney disease \& end-stage renal disease in the United States. Am J Kidney Dis. 2012;59(1 Suppl 1):A7-e420.

13 Institut canadien d'information sur la santé. Traitement du stade de l'insuffisance rénale organique au canada, de 2000 à 2009-Rapport annuel du registre canadien des insuffisances et des transplantations d'organes. Ottawa (Ont).ICIS.2011).

Retrieved from: http://publications.gc.ca/collections/collection_2011/icis-cihi/H11 5-38-2011-fra.pdf.

14 Modi GK, Jha V. The incidence of end-stage renal disease in India: a populationbased study. Kidney Int. 2006;70(12):2131-2133.

15 Boulahia Y. Urémie terminale traitée chez l'adulte dans la Wilaya d'Alger en 2004,2005 et 2006. PhD dissertation in medical sciences. Alger; 2009.

16 Awad SM. Chronic renal failure in Al-Anbar of Iraq. Saudi J Kidney Dis Transpl. 2011; 22(6):1280-1284.

17 Alalawi F, Ahmed M, AlNour H, Noralla M, Alhadari A. Epidemiology of end-stage renal disease in Dubai: single-center data. Saudi J Kidney Dis Transpl. 2017;28(5): 1119-1125.

18 Kao YY, Lee WC, Wang RH, Chen JB. Correlation of sociodemographic profiles with psychological problems among hospitalized patients receiving unplanned hemodialysis. Ren Fail. 2020;42(1):255-262.

19 Atkins RC. The epidemiology of chronic kidney disease. Kidney Int Suppl. 2005;(94): S14-S18.

20 Houat N. Incidence de l'insuffisance rénale chronique terminale à Maghnia, PhD dissertation in medical sciences. Tlemcen: Abou BekrBelkaid University; 2014-2015.

21 Asserraji M, Maoujoud O, Belarbi M, Oualim Z. Profil épidémiologique de l'insuffisance rénale terminale à l'hôpital Militaire de Rabat, Maroc [Epidemiological profile of end stage renaldisease at the Military Hospital in Rabat, Morocco]. Pan Afr Med J. 2015;20:439.

22 Moukeh G, Yacoub R, Fahdi F, Rastam S, Albitar S. Epidemiology of hemodialysis patients in Aleppo city. Saudi J Kidney Dis Transpl. 2009;20(1):140-146.

23 Bagnis CI, Couchoud C, Bowens M, et al. Epidemiology update for hepatitis C virus and hepatitis B virus in end stage renal disease in France. Liver Int. 2017;37:820-826.

24 Recommandations de Bonnes Pratiques Médicales Insuffisance Rénale Chronique Terminale société marocaine de néphrologie. Insuffisance Rénale Chronique Terminale. CIM 10: N 18. 2013. Retrieved from : http://nephro-maroc.com.

25 Afshar R, Sanavi S, Salimi J. Epidemiology of chronic renal failure in Iran: a four year single- center experience. Saudi J Kidney Dis Transpl. 2007;18(2):191-194.

26 Korgaonkar S, Tilea A, Gillespie BW, et al. Serum potassium and outcomes in CKD: insights from the RRI-CKD cohort study. Clin J Am Soc Nephrol. 2010;5(5):762-769.

27 Couchoud C, Kooman J, Finne P, et al. From registry data collection to international comparisons: examples of haemodialysis duration and frequency. Nephrol Dial Transplant. 2009;24(1):217-224.

28 Shigidi MM, Ramachandiran G, Rashed AH, Fituri OM. Demographic data and hemodialysis population dynamics in Qatar: a five year survey. Saudi J Kidney Dis Transpl. 2009;20(3):493-500. 